\title{
Application of Derivative Transform Spectroscopy in Gas Detection
}

\author{
Tingting Chen ${ }^{1}$, Chun Wang ${ }^{1}$, Jingqiu Liang ${ }^{2}$ and Jingsong $\mathrm{Li}^{1,2, *}$ \\ ${ }^{1}$ Laser Spectroscopy and Sensing Laboratory, Anhui University, 230601 Hefei, China \\ ${ }^{2}$ State Key Laboratory of Applied Optics, Changchun Institute of Optics, Fine Mechanics and Physics, \\ Chinese Academy of Sciences, Changchun 130033, China
}

\begin{abstract}
Digital filtering technique is of great significance in real-time signal processing and analysis, but the stability, efficiency and flexibility of filter algorithm are important indicators to reflect its application value. In this paper, an adaptive derivative transformation based on Savitzky-Golay filter algorithm was proposed for laser absorption spectroscopy analysis. To demonstrate this analysis algorithm, first-order and second-order derivative spectroscopy are evaluated for the analysis of infrared methane absorption spectra, and compared with the original direct absorption spectral signals. The results indicated that the proposed signal processing algorithm has good performance on noise suppression and spectral resolution improvement, and the 2nd derivative spectroscopy shows better de-noising efficiency.
\end{abstract}

\section{Introduction}

In recent years, many gas detection techniques have been developed, including physical and chemical detection methods [1-3]. Among of them, optical detection techniques based on laser spectroscopy, such as multiple-pass cell based tunable diode laser absorption spectroscopy, high precision optical cavity based cavity ring down spectroscopy and miniaturized acoustic signal detector based photoacoustic spectroscopy or quartz-enhanced photoacoustic spectroscopy and cantilever enhanced photoacoustic spectroscopy, are becoming more and more popular, mainly due to the advantages of fast response, high sensitivity and high resolution [4]. However, the realization of optical noise suppression (especially the annoying optical interference noise) to shot-noise limit is very challenging for optical spectroscopy in trace gases detection. To resolve this issue, various detection strategies and signal processing methods have been developed. Generally, it can be divided into hardware optimization and software algorithm design. As for the latter, it has significant advantages of low cost, convenient operation and high compatibility. To date, many advanced digital signal processing algorithms have been reported by combining with Wavelet Transform, Fourier transform, Wiener filter, Kalman filter and Savitzky-Golay filter, and successfully applied in laser spectroscopy sensor system for atmospheric greenhouse gas and trace gas monitoring and breathing gas diagnosis. However, digital filtering technique is of great significance in real-time signal processing and analysis, but

\footnotetext{
*Corresponding author: 1js0625@126.com
} 
the stability, efficiency and flexibility of filter algorithm are important indicators to reflect its application value.

Atmospheric methane is a typical greenhouse gas and trace gas, which plays more important affect on global warming. Therefore, it is of great significance and research value to develop highly sensitive methane detection technology. Methane has four significantly fundamental vibration modes, including two bending modes and two stretching modes, which makes it absorption spectrum mainly distributed in the infrared region. Based on the high-resolution transmission molecular absorption database HITRAN [5], the spectral distribution characteristic of methane absorption line strengths in the whole infrared region has been simulated and shown in Figure 1. In laser absorption spectroscopy, the spectral cross-section absorption interference is also a key factor that affects the accuracy of measurement results, including self and non-target absorption interference. For this issue, the related research results show that derivative spectroscopy is an effective method to improve spectral resolution.

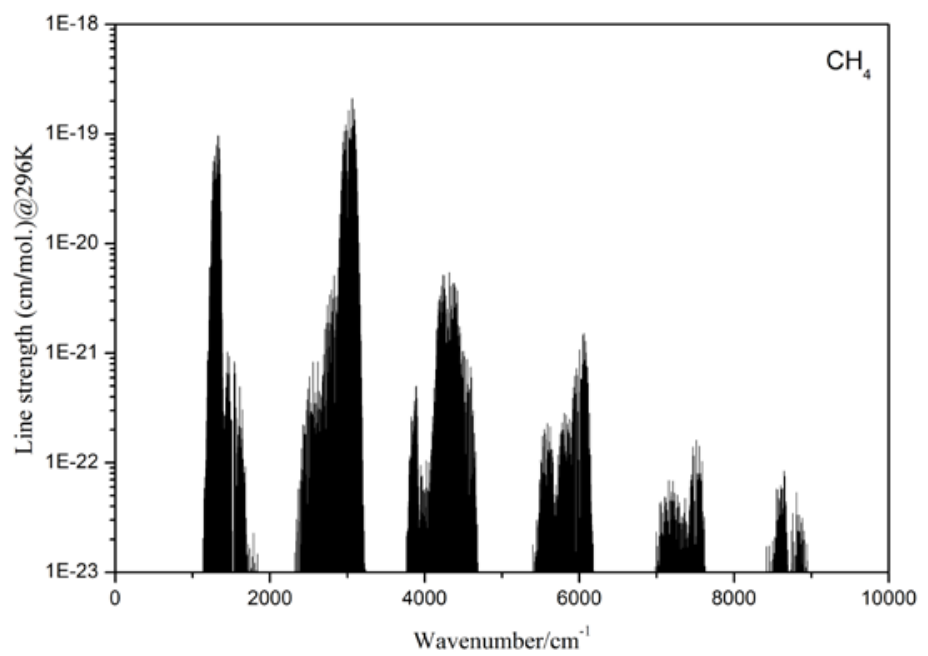

Fig. 1. Methane spectral line distribution characteristics

In this paper, an adaptive derivative transformation spectroscopy technique based on Savitzky-Golay filter algorithm was proposed for simultaneous noise suppression and spectral resolution improvement. To demonstrate this technique, primary performance on methane infrared absorption spectra was investigated in detail.

\section{Basic theory and algorithm}

\subsection{Lambert-Beer law}

The fundamental theory of laser absorption spectroscopy has been detailedly described in many literature, therefore, only the basic principles are reviewed here for spectral simulation. The absorption process between light and absorb medium can be described by the well known Lambert-Beer relation:

$$
I(v)=I_{0}(v) \exp (-\alpha(v) L)
$$


where $I(v)$ and $I_{0}(v)$ are the incident light intensity and the transmitted light intensity, respectively. $L$ is the effective optical path of the interaction between light and molecule. $\alpha(v)=\phi\left(v-v_{0}\right) \cdot S(T) \cdot N(T, P)$ is the molecular absorption coefficient at frequency $v\left(\mathrm{~cm}^{-1}\right)$, which is a function of molecular absorption lineshape $\phi\left(v-v_{0}\right)$, temperature dependent (T) line strength $S(T)$ and the number density of the target molecule $N(T, P)$. According to the broadening mechanism, there are three types of absorption lineshapes commonly used in absorption spectroscopy, i.e. Gaussian profile (at lower pressure), a Lorentzian profile (at higher pressure), and a Voigt profile (at intermediate cases), which are adaptive to the normalization condition. Hereby, the integral absorption area $(A)$ can be expressed as:

$$
A=\int_{-\infty}^{\infty} \alpha(v) L d v=\int_{-\infty}^{\infty} \phi\left(v-v_{0}\right) \cdot S(T) \cdot N(T, P) \cdot L d v=S(T) \cdot N(T, P) \cdot L(2)
$$

The molecular absorption line intensity can be directly taken from the HITRAN database. with the known experimental conditions of temperature $\mathrm{T}$, pressure $\mathrm{P}$ and the effective optical L, the molecule number density or concentration can be calculated by combining with the ideal gas state equation.

\subsection{Savitzky-Golay filter}

The Savitzky-Golay (S-G) filtering algorithm is a data stream smoothing de-noising filtering method first proposed by Savitzky and Golay in 1964 [6]. Unlike the traditional moving window averaging method, it performs a least square fitting procedure. The idea of S-G filtering is to find filter coefficients within the moving window not by a constant, but by a higher order polynomial. This algorithm has been proved without inducing signal broadening and suffering loss of signal amplitude. In this study, the algorithm has been improved with derivative transformation, except the de-noising process, which can be mathematically described as

$$
f_{t}^{n, s}=\sum_{i=-m}^{m} h_{i}^{n, s, t} y_{i}
$$

where $\mathrm{n}$ is the Polynomial order, $2 \mathrm{~m}+1$ is total sampling points, $\mathrm{s}=0$ for de-noising process, $\mathrm{s}>0$ for derivative transformation, $h_{i}^{n, s, t}$ is the convolution weight coefficient of the ith point when evaluating the s-order differential at the point $t$, which can be expressed as

$$
h_{i}^{n, s, t}=\sum_{k=0}^{n} \frac{(2 k+1)(2 m)^{(k)}}{(2 m+k+1)^{(k+1)}} P_{k}^{m}(i) P_{k}^{m, s}(t)
$$

where $(2 \mathrm{~m})^{(k)}$ the generalized factorial functions, $P_{k}^{m}(i)$ is the Gram polynomial function, which is defined as: 


$$
P_{k}^{m}(i)=\sum_{j=0}^{k} \frac{(-1)^{j+k}(j+k)^{(2 j)}(m+t)^{(j)}}{(j !)^{2}(2 m)^{(j)}}
$$

Therefore, the S-G filtering model established here can simultaneously realize the de-noising processing and derivative calculation. The signal-to-noise ratio (SNR) of the spectrum can be improved through the filtering and de-noising processing, while the derivative processing is conducive to improving the spectral resolution.

Table 1. Spectroscopic parameters for the $\mathrm{R}_{3}$ transitions of $\mathrm{CH}_{4}$ near $6057 \mathrm{~cm}^{-1}$.

\begin{tabular}{c|c|c|c}
\hline $\begin{array}{c}\text { Position } \\
\left(\mathrm{cm}^{-1}\right)\end{array}$ & $\begin{array}{c}\text { Line intensity } \\
(\mathrm{cm}-1 / \mathrm{mol} . @ 296 \mathrm{~K})\end{array}$ & $\begin{array}{c}\text { Air-broadening coefficient } \\
\left(\mathrm{cm}^{-1} / \mathrm{atm}\right)\end{array}$ & $\begin{array}{c}\text { Self-broadening coefficient } \\
\left(\mathrm{cm}^{-1} / \mathrm{atm}\right)\end{array}$ \\
\hline 6057.0861 & $1.278 \mathrm{E}-21$ & 0.0672 & 0.083 \\
\hline 6057.0998 & $9.061 \mathrm{E}-22$ & 0.0536 & 0.083 \\
\hline 6057.1273 & $8.694 \mathrm{E}-22$ & 0.0560 & 0.083 \\
\hline
\end{tabular}

\section{Result and discussion}

A home-made computer program was written for evaluating the developed filter algorithm. Three adjacent methane absorption line transitions near $6057 \mathrm{~cm}^{-1}$ were specially selected to test the derivative transform for resolution enhancement. The related spectroscopic parameters are summarized in Table 1 . Base on the theory mentioned above, the simulated $\mathrm{CH}_{4}$ absorption spectrum between $6056-6058 \mathrm{~cm}^{-1}$ are shown in Figure 2 with different sample pressure. As can see, the absorption spectra have been unresolved with increasing sample pressure.

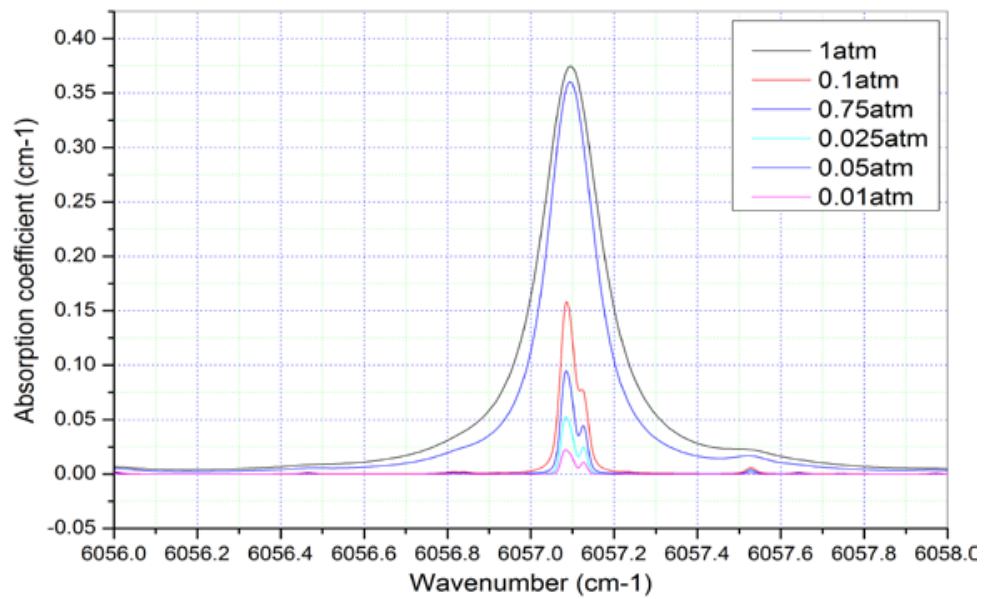

Fig. 2. Simulated $\mathrm{CH}_{4}$ absorption spectra with different sample pressures 


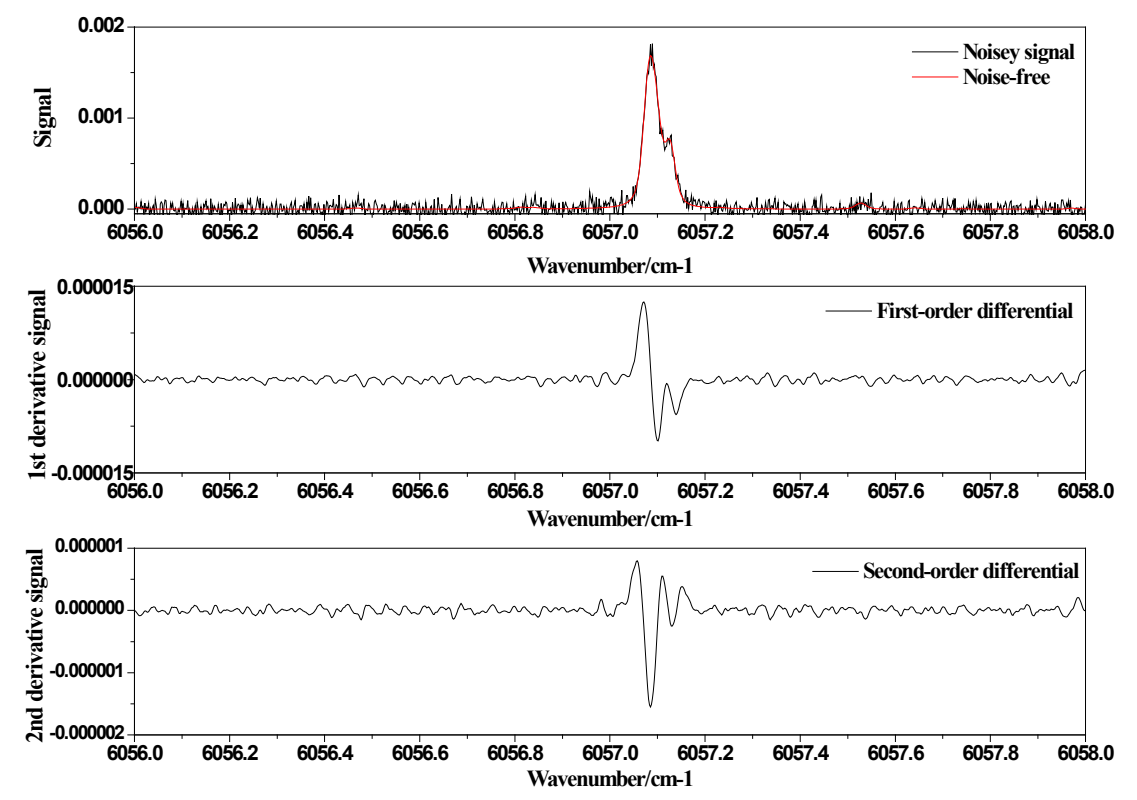

Fig. 3. Spectral simulation of the noise-free $\mathrm{CH}_{4}$ absorption spectrum and synthetic noisy spectral signal (Upper panel), and the S-G filtered spectra with 1st derivative signal (Middle panel) and 2nd derivative signal (Lower panel).

To evaluate the performance of the S-G filter based signal de-noising and derivative calculation, lots of spectra were simulated and purposefully corrupted with Gaussian white noise to simulate real signals. For example, figure 3 illustrated a typical $\mathrm{CH}_{4}$ absorption spectrum, white noise, the synthetic noisy signal, and after the application of S-G filter de-noising with different filter parameters, as well as the first-order and second-order derivative spectra of the S-G filtered spectrum with the optimal filter parameters. From this figure, we can see that the spectral SNR by the S-G filter de-noising has been significantly improved. In this case, the optimal S-G filter parameters with polynomial order of 3 and filter window width of 25 has been applied to the spectral data. By adopting this pair of optimal filtering parameters, the filtered signal has been further differentiated to yield the 1st and 2nd derivative signal. Two absorption peaks can be obviously observed in the derivative spectral signal, and the signal amplitude decreases with the increase of the order of derivative transform, which is consistent with the theoretical expectation. According to the derivative principle, the peak of 2 nd derivative signal should be consistent with the center position of the molecular absorption line. Therefore, we can conclude that the strongest negative absorption peak on the left side corresponds to $\mathrm{CH}_{4}$ absorption line at $6057.0861 \mathrm{~cm}^{-1}$, while the relatively weak negative peak on the right side corresponds to another $\mathrm{CH}_{4}$ absorption line at $6057.13 \mathrm{~cm}^{-1}$. Note that the slight asymmetry of positive peaks mainly attributes to the contribution of $\mathrm{CH}_{4}$ absorption line near $6057.10 \mathrm{~cm}^{-1}$. Since the frequency spacing between the first two-line is only $0.0137 \mathrm{~cm}^{-1}$, thus it is very challenging to distinguish the triple merger absorption lines, even at the limit of Doppler broadening conditions. 

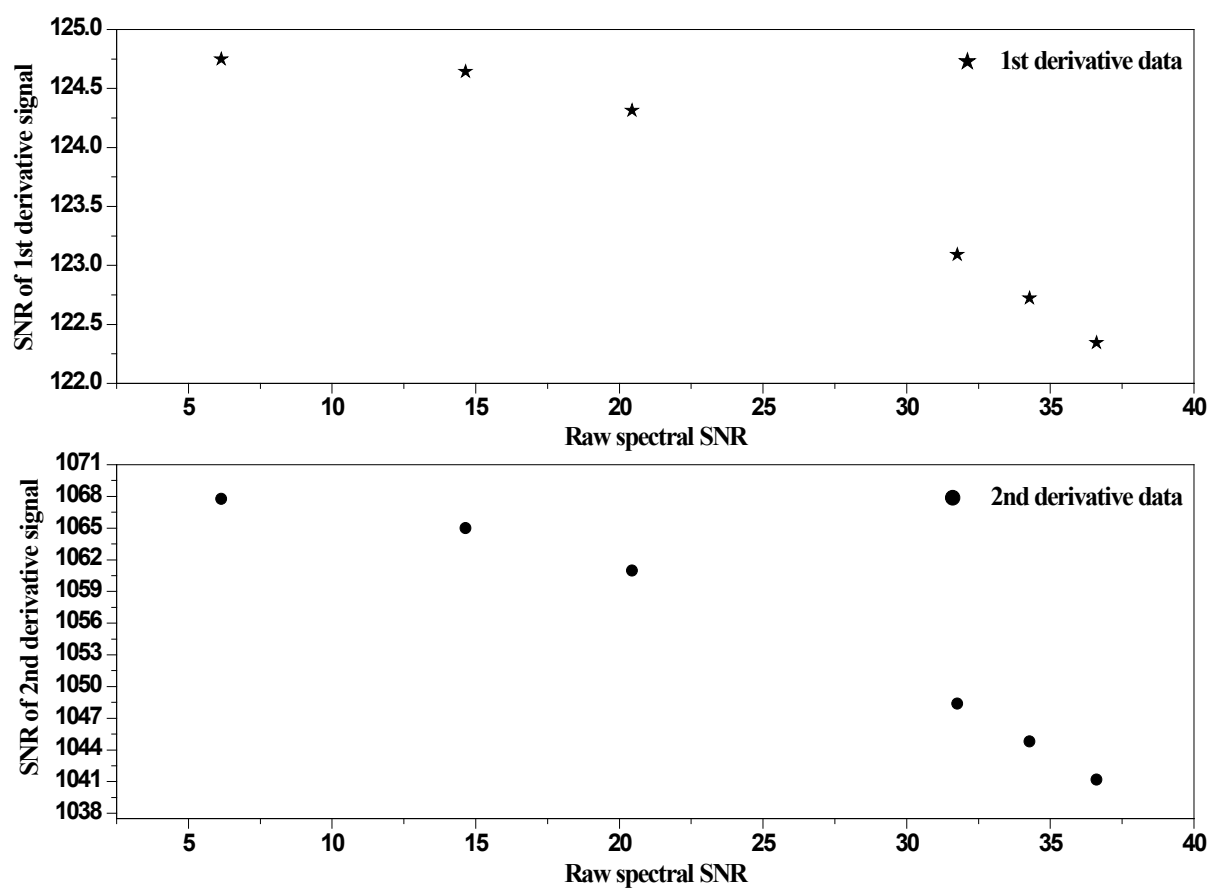

Fig. 4. SNR comparison of raw spectral and derivative spectra

According to a similar procedure, figure 4 summarizes other spectral results and the comparison of SNR enhancement before and after application of the S-G filter algorithm. On the whole, the 2nd derivative transform shows better performance than the $1 \mathrm{st}$ derivative transform. In the case of wavelength modulation spectroscopy (WMS), the second harmonic (2F) signal are commonly used for retrieving gas concentration, due to its linear response to sample gas concentration. Based on this ideal, the relationship between the signal amplitude of 2 nd derivative spectra and the absorbed molecular concentration has investigated. Because of the limitation of original spectral resolution, the characteristics of two absorption peaks can only be distinguished near $6057.09 \mathrm{~cm}^{-1}$ and $6057.13 \mathrm{~cm}^{-1}$ in this study.

The peak-to-peak values for the distinguishable spectral lines are plotted as a function of $\mathrm{CH}_{4}$ concentration are shown in figure 5. Linear regression algorithm was used to fit the experimental data as shown in the insert. Finally, the calculated linear regression coefficient $\mathrm{R}^{2}$ values were 0.9999 for absorption line at $6057.09 \mathrm{~cm}^{-1}$ and $\sim 1.0$ for absorption line at $6057.13 \mathrm{~cm}^{-1}$. This finding exhibits an excellent linearity between the peak-to-peak values and $\mathrm{CH}_{4}$ concentrations. Similar to WMS-2F detection method, the calibration equation in 2nd derivative transform spectroscopy can also be used for trace gas concentration measurement. 

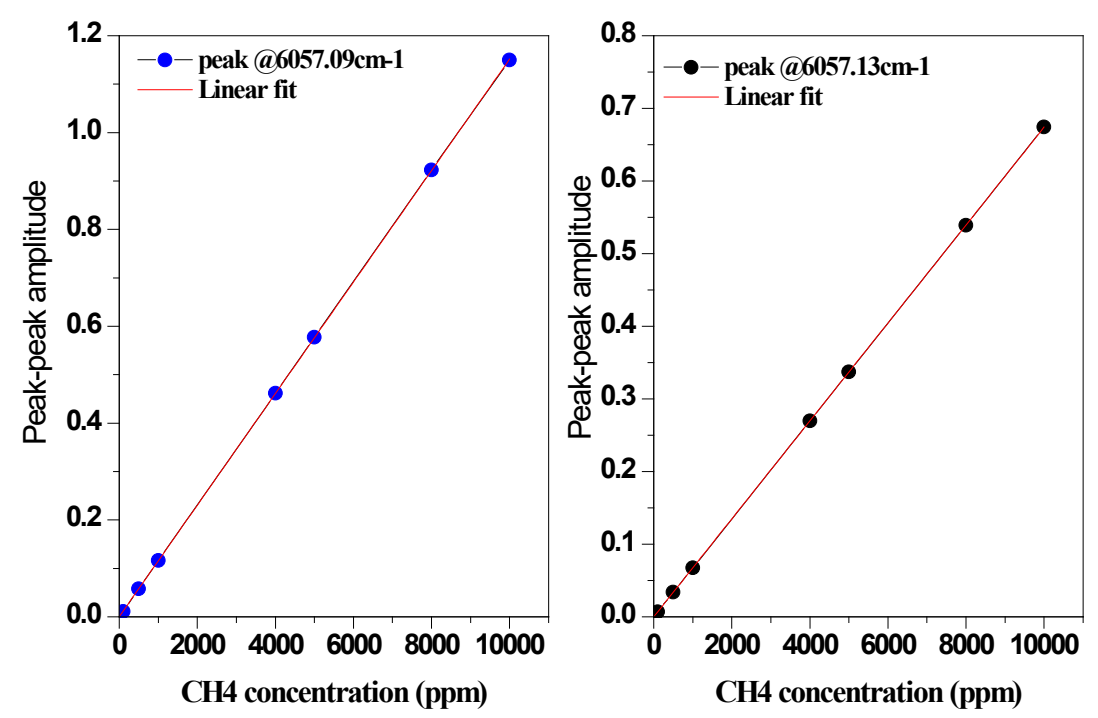

Fig. 5. The peak-to-peak value of $2^{\text {nd }}$ derivative spectra vs. $\mathrm{CH}_{4}$ concentration, and the linear fits

\section{Conclusion}

In this paper, an adaptive derivative transformation spectroscopy based on Savitzky-Golay filter algorithm was proposed for laser absorption spectroscopy analysis. To demonstrate this analysis algorithm, 1st and 2nd derivative spectroscopy are evaluated for the analysis of multi-merger $\mathrm{CH}_{4}$ absorption spectra. Primary results show that the proposed signal processing algorithm has good performance on noise suppression and spectral resolution improvement. In comparison, the 2nd derivative spectroscopy shows slightly better de-noising efficiency than the 1 st derivative spectroscopy, and the results show that it has similar characteristics of the well-known WMS-2F detection technique, which demonstrates its great advantages and development prospects for trace gases detection $[7,8]$.

\section{Acknowledgments}

The authors gratefully acknowledge the financial support from the National Natural Science Foundation of China (41875158), the financial support from the Open Fund of State Key Laboratory of Applied Optics (SKLAO2020001A13), and Innovation Training Program and Education Reform of Anhui University (Y020412012/006, 2020jyxm0100, Y020412003/056, Y020712002/245).

\section{References}

[1] Schwaighofer A, Brandstetter M, Lendl B. Quantum cascade lasers (QCLs) in biomedical spectroscopy[J]. Chemical Society Reviews, 46:5903-5924,2017.

[2] Li J, Chen W, Fischer H. Quantum cascade laser spectrometry techniques: a new trend in atmospheric chemistry. Applied Spectroscopy Reviews 48 :523-559, 2013.

[3] Zhang L, Tian G, Li J, Yu B. Applications of absorption spectroscopy using quantum cascade lasers[J]. Applied Spectroscopy 68(10): 1095-1107,2014. 
[4] Li J, Yu B, Zhao W, Chen W. A review of signal enhancement and noise reduction techniques for Tunable Diode Laser Absorption Spectroscopy[J]. Applied Spectroscopy Reviews 49:666-691, 2014.

[5] Gordon I E, Rothman L S, Hill C, et al. The HITRAN 2016 molecular spectroscopic database[J]. J. Quant. Spectrosc. Radiat. Transfer 203:3-69,2017.

[6] Press W H, Teukolsky S A. Savitzky-Golay smoothing filters[J]. Computers in Physics, 4(6): 669-672,1990.

[7] Nwaboh J A, Desbois T, Romanini D, et al. Molecular Laser Spectroscopy as a Tool for Gas Analysis Applications[J]. International Journal of Spectroscopy, 2011:1-12,2011.

[8] Nagapriya K S, Sinha S, Prashanth P, et al. Laser Calorimetry Spectroscopy for ppm-level Dissolved Gas Detection and Analysis[J]. Scientific Reports, 7:42917, 2017. 